\title{
Radio Emission from Low-Luminosity Active Galactic Nuclei
}

Schuyler D. Van Dyk ${ }^{1}$

Dept. Phys $\mathcal{E}^{\circ}$ Astr., UCLA, Los Angeles, CA 90095, U.S.A.

Luis C. Ho

Harvard-Smithsonian Center for Astrophysics, Cambridge, MA 02138, U.S.A.

\begin{abstract}
Many nearby galaxies show optical evidence for low-luminosity AGNs that are far less luminous than classical Seyfert nuclei and QSOs. LINERs, the most common variety of such emission-line objects, comprise $\sim 1 / 3$ of nearby galaxies, and may serve as an important "missing link" between normal and Seyfert galaxies. To shed light on the physical origin of LINERs, which remains controversial, we are conducting a high-resolution continuum survey of a representative sample of galaxies using the VLA to search for compact radio cores. We additionally discuss the dramatic radio variability in the LINER nucleus of M81.
\end{abstract}

\section{The Nature of LINERs and the Need for Radio Observations}

A longstanding controversy over low-ionization nuclear emission-line regions (LINERs; Heckman 1980), concerns their physical origin. Based on the close resemblance of some LINERs to Seyfert nuclei, LINERs may be another manifestation of the AGN phenomenon, as accretion onto a central supermassive black hole provides the energy. An alternative view is that stellar processes (photoionization by hot stars, heating by shocks) account for the energy. Since the optical line luminosities of LINERs are meager, the energetics alone do not provide a useful discriminator between the two possible mechanisms (see Filippenko 1996 for a review). At least some LINERs are simply extensions of the Seyfert phenomenon, based on the presence of an ionizing UV continuum, broad $\mathrm{H} \alpha$ line, or hard X-rays. Unknown is the fraction of LINERs that are actually AGNs, which has important ramifications for the very poorly constrained faint end of the AGN luminosity function.

High-resolution, multi-frequency radio continuum observations can additionally serve to diagnose LINER nuclei. Past studies of nearby galaxies using the VLA show that LINERs may have steep spectra (Heckman et al. 1983; Keel 1984). However, these studies were done with fairly low sensitivity at low frequencies. Higher frequency observations provide higher angular resolution, putting tighter constraints on a central source, more stringent limits on the brightness temperature, resolving out extended nuclear structures, and detailing thermal versus nonthermal emission. We have obtained high-resolution ( $A$ and $B$ array) radio continuum VLA data at 3.6 and $6 \mathrm{~cm}$ for a statistically meaningful sample $(\sim 34)$ of LINERs selected from the optical spectroscopic survey of Ho et al. $(1995,1997)$. Weak ( $\sim$ few mJy), compact $\left(<1^{\prime \prime}\right)$ radio emission is evident from many of the sources.

\footnotetext{
${ }^{1}$ Visiting scientist.
} 


\section{The Case of the M81 Nucleus}

The nearby galaxy M81 contains a well known LINER nucleus which is a compact, flat-spectrum, variable radio core (Bietenholz, Bartel, \& Rupen, these Proceedings, p. 201). From the VLA monitoring of SN 1993J (Van Dyk et al., these Proceedings, p. 357), we have obtained radio light curves for the M81 nucleus shown in Figure 1. The nucleus shows distinct variability, particularly at $3.6 \mathrm{~cm}$. The largest "flare" occurs first at $3.6 \mathrm{~cm}$, and then at the lower frequencies. The data shown represent the best multi-frequency monitoring yet of a LINER. Ho et al. (1996) found no flux variations greater than $20 \%$ in the broad $\mathrm{H} \alpha$ line between 1984 and 1993. However, in $1995 \mathrm{March}$, Bower et al. (1996) found in their $H S T$ spectra a new double-peaked broad component in the line profile. The radio galaxy Pictor A (Halpern \& Eracleous 1994) and, especially, the LINER NGC 1097 (Storchi-Bergmann et al. 1995) have shown similar double-peaked lines, which may originate from emission from the outer parts of an accretion disk or from the ends of a jet emerging from the center. The radio flares in M81 may be directly associated with the emergence of the double-peaked $\mathrm{H} \alpha$ line, and both transient events are likely related to a sudden increase in the accretion rate.

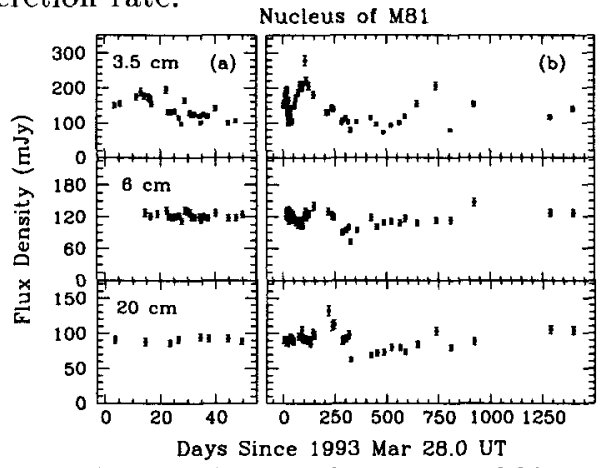

Figure 1. Flux density vs. time for the nucleus of $M 81$ at $3.6,6$, and $20 \mathrm{~cm}$. The nucleus shows distinct variability, especially at $3.6 \mathrm{~cm}$. The left panel shows an expanded view of the first 55 days.

\section{References}

Bower, G. A., et al. 1996. AJ, 111, 1901-1907.

Filippenko, A. V. 1996. In The Physics of Liners in View of Recent Observations, eds. M. Eracleous et al., ASP Conf. Series, 103, 17-43.

Halpern, J. P., \& Eracleous, M. 1994. ApJ, 433, L17-20.

Heckman, T. M. 1980. $A 6 A, 87,152-164$.

Heckman, T. M., et al. 1983. AJ, 88, 1077-1087.

Ho, L. C., Filippenko, A. V., \& Sargent, W. L. W. 1995. ApJS, 98, 477-593.

Ho, L. C., Filippenko, A. V., \& Sargent, W. L. W. 1996. ApJ, 462, 183-202.

Ho, L.C., Filippenko, A. V., \& Sargent, W. L. W. 1997. ApJS, 112, 315-390.

Keel, W. C. 1983. ApJ, 269, 466-486.

Keel, W. C. 1984. $A p J, \mathbf{2 8 2}, 75-84$.

Storchi-Bergmann, T., et al. 1995, ApJ, 443, 617-624. 